\title{
Some complexity results in topology and analysis
}

\author{
by
}

\author{
Steve Jackson $\dagger$ and R. Daniel Mauldin $\ddagger$ (Denton, Tex.)
}

\begin{abstract}
If $X$ is a compact metric space of dimension $n$, then $K(X)$, the $n$ dimensional kernel of $X$, is the union of all $n$-dimensional Cantor manifolds in $X$. Aleksandrov raised the problem of what the descriptive complexity of $K(X)$ could be. A straightforward analysis shows that if $X$ is an $n$-dimensional complete separable metric space, then $K(X)$ is a $\Sigma_{2}^{1}$ or PCA set. We show (a) there is an $n$-dimensional continuum $X$ in $\mathbb{R}^{n+1}$ for which $K(X)$ is a complete $\boldsymbol{\Pi}_{1}^{1}$ set. In particular, $K(X) \in \boldsymbol{\Pi}_{1}^{1}-\boldsymbol{\Sigma}_{1}^{1} ; K(X)$ is coanalytic but is not an analytic set and (b) there is an $n$-dimensional continuum $X$ in $\mathbb{R}^{n+2}$ for which $K(X)$ is a complete $\boldsymbol{\Sigma}_{2}^{1}$ set. In particular, $K(X) \in \boldsymbol{\Sigma}_{2}^{1}-\boldsymbol{\Pi}_{2}^{1} ; K(X)$ is PCA, but not CPCA. It is also shown the Lebesgue measure as a function on the closed subsets of $[0,1]$ is an explicit example of an upper semicontinuous function which is not countably continuous.
\end{abstract}

I. Complexity of $K(X)$. Let us recall that an $n$-dimensional Cantor manifold is a compact $n$-dimensional space which cannot be separated by a closed subset of dimension $\leq n-2$. Since there are complete separable metric spaces of dimension $n$ which contain no compact sets of dimension $n$, there are $n$-dimensional spaces which contain no Cantor manifolds of dimension $n$. However, every compact metric space of dimension $n$ does contain an $n$-dimensional Cantor manifold. If $X$ is a compact metric space of dimension $n$, then $K(X)$, the $n$-dimensional kernel of $X$, is the union of all $n$-dimensional Cantor manifolds in $X$. R. Pol $[\mathrm{P}]$, in answering a question of Aleksandrov [A], has given an example of a space $X$ such that $X \backslash K(X)$ is still $n$-dimensional. As Pol pointed out to us, Aleksandrov also raised the question of what the descriptive complexity of $K(X)$ could be. For Pol's example, it turns out that $K(X)$ is a Borel set. We will determine the exact bounds on the complexity of $K(X)$ in this paper. We first note the following

1991 Mathematics Subject Classification: Primary 54B05, 04A05; Secondary 28A05.

Key words and phrases: Cantor manifold, dimensional kernel, projective set, countably continuous, upper semicontinuous.

$\dagger$ Supported by National Science Foundation grant DMS 9007808.

$\ddagger$ Supported by National Science Foundation grant DMS 9007035. 
simple bound on the complexity of $K(X)$.

TheOREM 1. Let $X$ be an $n$-dimensional complete separable metric space. Then $K(X)$ is a $\Sigma_{2}^{1}$ or PCA set.

Pr o of. Let $C$ be the set of all Cantor manifolds in the space, and $\mathcal{K}(X)$, of all compact subsets of $X$. Then $K(X)=\operatorname{proj}_{1}\{(x, M): x \in M$ and $M \in C\}$. Also, $\mathcal{K}(X) \backslash C=\operatorname{proj}_{1}\{(M, L) \in \mathcal{K}(X) \times \mathcal{K}(X): \operatorname{dim} L \leq n-2$ and $L$ separates $M$ \}. Clearly, this last set of pairs is an analytic set. Thus, $C$ is a coanalytic or $\boldsymbol{\Pi}_{1}^{1}$ set and $K(X)$ is a PCA set.

Our next theorem is to show that this simple estimate is fairly sharp. We assume all our spaces $X$ are compact.

Theorem 2. Let $n \geq 2$.

(a) If $X \subset \mathbb{R}^{n}$ and $\operatorname{dim} X=n$, then $K(X)$ is an $F_{\sigma}$ set.

(b) There is an $n$-dimensional compact set $X$ in $\mathbb{R}^{n+1}$ for which $K(X)$ is a complete $\boldsymbol{\Pi}_{1}^{1}$ set. In particular, $K(X) \in \boldsymbol{\Pi}_{1}^{1}-\boldsymbol{\Sigma}_{1}^{1} ; K(X)$ is coanalytic but is not an analytic set.

(c) There is an $n$-dimensional compact set $X$ in $\mathbb{R}^{n+2}$ for which $K(X)$ is a complete $\boldsymbol{\Sigma}_{2}^{1}$ set. In particular, $K(X) \in \boldsymbol{\Sigma}_{2}^{1}-\boldsymbol{\Pi}_{2}^{1} ; K(X)$ is PCA, but not $C P C A$.

Remarks. Parts (a) and (c) of this theorem are the best possible. We do not know whether part (b) can be improved to $\boldsymbol{\Sigma}_{2}^{1}$ as well. Also, we could easily make the sets constructed for parts (b) and (c) continua.

Proof. For the proof of (a), let us recall that every compact $n$-dimensional Cantor manifold in $X$ lies in a maximal Cantor manifold in $X$, a dimensional component of $X$ [E, p. 99]. Since every $n$-dimensional subset of $\mathbb{R}^{n}$ contains an open set and no two dimensional components of $X$ can have interior points in common, $X$ has only countably many dimensional components. Thus, $K(X)$ is an $F_{\sigma}$ set.

To prove parts (b) and (c), we assume for simplicity that $n=2$. The general case differs little from the arguments below.

To prove (b), let $B=\{(0,0, z): z \in C\}$, where $C$ is the middle third Cantor set. Let $P \subset B$ be $\boldsymbol{\Pi}_{1}^{1}$. We will produce a compactum $X \subset \mathbb{R}^{3}$ such that $K(X) \cap B=P$. This easily gives that $K(X)$ is not necessarily a Borel set and that $K(X)$ may be $\boldsymbol{\Pi}_{1}^{1}$ complete. We will define $X$ by defining its "sections", $X_{z}=\{(x, y):(x, y, z) \in X\}$, for each $z \in C$.

Suppose now that $z \in C$. We first construct a set $D_{z}$, "a framework", which will always be a subset of $X_{z}$. Figure 1a illustrates this framework. We begin with the triangle which is the union of the line segments joining $(0,0, z),(1,1, z)$ and $(-1,1, z)$. We then adjoin the line segments $L_{k}$ from $\left(1-1 / 2^{k}, 1, z\right)$ to $(0,0, z)$, for $k=0,1,2, \ldots$ We next add a series of 
2-dimensional "tubes" as follows. We first add tubes $T(1), T(2), T(3), \ldots$ where $T(i)$ is the intersection of the above triangular region with the rectangular region $\left\{(x, y, z):|x| \leq 1\right.$ and $\left.\left|y-1 / 2^{i}\right|<1 / 4 \cdot 2^{i}\right\}$. For convenience, we also set $T(0)$ to be the top edge of the triangular region. The top edge is the only tube which is not 2 -dimensional. We then add tubes $T(i, j)$ as follows: first $T(i, 0)=T(i)$ and if $j \neq 0$, then:

(a) $T(i, j)$ is the intersection of a rectangular region with the triangular region formed by the line segment $L_{0}$ and the top and right edges of the original triangular region.

(b) Each $T(i, j)$ lies strictly between $T(i)$ and $T(i+1)$.

(c) $T(i, j)$ lies above $T(i, j+1)$.

(d) $T(i, j) \cap T\left(i^{\prime}, j^{\prime}\right)=\emptyset$, for $(i, j) \neq\left(i^{\prime}, j^{\prime}\right)$.

(e) For each $i$, the only limit points of $\bigcup_{j} T(i, j)$ not in $\bigcup_{j} T(i, j)$ lie on $T(i+1)$.

In general, we construct tubes $T\left(i_{1}, \ldots, i_{n}\right)$ for all $\left(i_{1}, \ldots, i_{n}\right) \in \omega^{<\omega}$ such that $T\left(i_{1}, \ldots, i_{n-1}, 0\right)=T\left(i_{1}, \ldots, i_{n-1}\right)$ and for $i_{n} \neq 0$ satisfying the analogous requirements:

(a) $T\left(i_{1}, \ldots, i_{n}\right)$ is the intersection of a rectangular region with the triangular region formed by the line segment $L_{n-2}$ and the top and right edges of the original triangular region.

(b) Each $T\left(i_{1}, \ldots, i_{n}\right)$ lies strictly between $T\left(i_{1}, \ldots, i_{n-1}\right)$ and $T\left(i_{1}, \ldots, i_{n-1}+1\right)$.

(c) $T\left(i_{1}, \ldots, i_{n}\right)$ lies above $T\left(i_{1}, \ldots, i_{n}+1\right)$.

(d) $T\left(i_{1}, \ldots, i_{n}\right) \cap T\left(i_{1}^{\prime}, \ldots, i_{n}^{\prime}\right)=\emptyset$, for $\left(i_{1}, \ldots, i_{n}\right) \neq\left(i_{1}^{\prime}, \ldots, i_{n}^{\prime}\right)$.

(e) For each $\left(i_{1}, \ldots, i_{n-1}\right)$, the only limit points of $\bigcup_{j} T\left(i_{1}, \ldots, i_{n-1}, j\right)$ not in $\bigcup_{j} T\left(i_{1}, \ldots, i_{n-1}, j\right)$ lie on $T\left(i_{1}, \ldots, i_{n-1}+1\right)$.

The framework $D_{z}$ is completed by adding all the sets $T\left(i_{1}, \ldots, i_{n}\right)$ to those already adjoined.

Let $P \subset B$ be $\boldsymbol{\Pi}_{1}^{1}$ and let $T$ be a tree on $\{0,1\} \times \omega$ which codes $P$. In other words, $T \subset \bigcup_{n=0}^{\infty}\{0,1\}^{n} \times \omega^{n}$ with the property that $(r, s) \in T \rightarrow$ $(r|k, s| k) \in T$, for all $k<\ln (r)$ and $T$ is such that for all $z \in\{0,1\}^{\omega}(\simeq C)$ we have $(0,0, z) \in P \leftrightarrow T_{z}$ is well-founded, where $T_{z}=\left\{s \in \omega^{<\omega}:(z \mid \ln (s), s)\right.$ $\in T\}$.

For a fixed $z \in\{0,1\}^{\omega}$, we obtain $X_{z}$ by adjoining to $D_{z}$ certain regions as follows:

Let $f: \omega \rightarrow \omega$ be an onto map such that $f^{-1}(n)$ is infinite for all $n$. For each $\left(i_{1}, \ldots, i_{n}\right)$ such that $\left(f\left(i_{1}\right), \ldots, f\left(i_{n}\right)\right) \notin T_{z}$ we add to $D_{z}$ the open region $R\left(i_{1}, \ldots, i_{n}\right)$ which is bounded by the line segment $L_{n-2}$ and the line segment from $(0,0, z)$ to $(1,1, z)$ (where $L_{-1}$ is defined to be the left hand boundary of the triangular region) and by the tubes $T\left(i_{1}, \ldots, i_{n}\right)$ and 
$T\left(i_{1}, \ldots, i_{n}+1\right)$. Of course, the boundary of this region is already in $X_{z}$.

This second stage is illustrated in Fig. 1b.

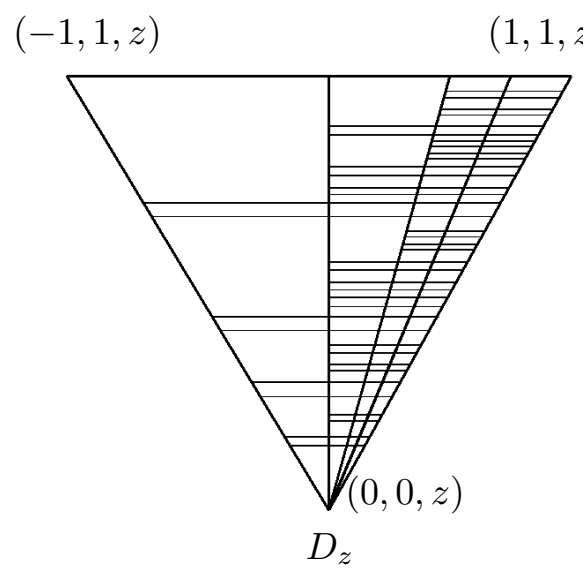

Fig. 1a. The framework

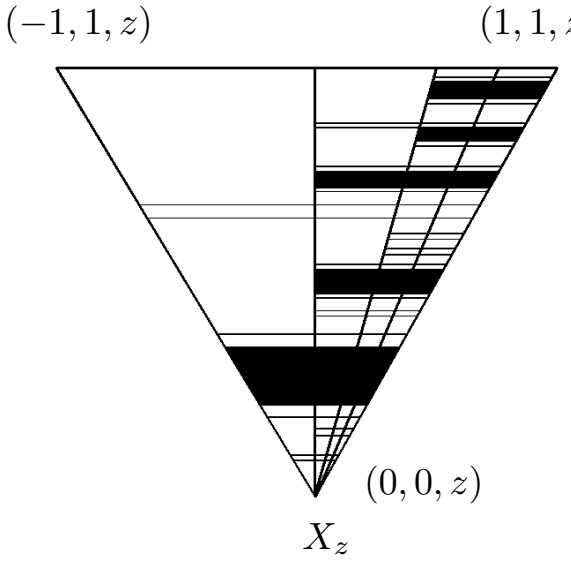

Fig. 1b. Adjoining regions

This completes the definition of $X_{z}$ and hence of $X$. Since $X \subset \mathbb{R}^{2} \times C$, $\operatorname{dim} X \leq 2$ and since the sections of $X$ are 2-dimensional, $\operatorname{dim} X=2$.

Clearly, each section $X_{z}$ of $X$ is closed. In order to see that $X$ itself is closed, let $\left\{\left(x_{n}, y_{n}, z_{n}\right)\right\}_{n=1}^{\infty}$ be a sequence of points of $X$ converging to a point $p=(x, y, z)$. If $(x, y, z) \in D_{z}$, then $p \in X$. So, suppose $p$ lies in some open region $R^{z}\left(i_{1}, \ldots, i_{m}\right)$. For $n$ sufficiently large, $\left(x_{n}, y_{n}, z_{n}\right) \in$ $R^{z_{n}}\left(i_{1}, \ldots, i_{m}\right)$. This implies that for $n$ sufficiently large, $\left(f\left(i_{1}\right), \ldots, f\left(i_{m}\right)\right)$ $\notin T_{z_{n}}$. Since $z_{n} \rightarrow z$ in $\{0,1\}^{\omega}$, we have $\left(f\left(i_{1}\right), \ldots, f\left(i_{m}\right)\right) \notin T_{z}$. This implies $(x, y, z) \in X_{z}$ and $X$ is closed.

We now show $(0,0, z) \in P \leftrightarrow(0,0, z) \in K(X)$. Note that if $M$ is a Cantor manifold lying in $X$ then $M$ must be a subset of some section $X_{z}$, since any two sections of $X$ can be separated from each other by a zerodimensional set - namely the empty set.

First, assume $(0,0, z) \notin P$. The tree $T_{z}$ is ill-founded meaning there is a sequence $\left(j_{1}, j_{2}, \ldots\right) \in\{0,1\}^{\omega}$ such that $\left(z \mid m,\left(j_{1}, \ldots, j_{m}\right)\right) \in T$ for all $m$. Thus, for any $\left(i_{1}, \ldots, i_{m}\right)$ such that $f\left(i_{1}\right)=j_{1}, \ldots, f\left(i_{m}\right)=j_{m}$, the portion of the open region $R\left(i_{1}, \ldots, i_{m}\right)$ lying between $L_{m-2}$ and $L_{m-1}$ is disjoint from $X_{z}$. However, by removing countably many points from $X_{z}$ (along each line $L_{k}$ and the left and right edges of the triangular region), we may then clearly disconnect $X_{z}$ by a countable set. Since the sets $f^{-1}\left(j_{q}\right)$ are infinite, we may, in fact, disconnect $X_{z}$ by a countable set $C$ so that $X_{z} \backslash C=U \cup V$ where $V \subset B_{\varepsilon}(0,0, z)$, for $\varepsilon$ arbitrarily small. Thus, there is no Cantor manifold $M$ lying in $X$ and containing $(0,0, z)$. So, $(0,0, z) \notin P$ implies $(0,0, z) \notin K(X)$.

Assume now that $(0,0, z) \in P$, or equivalently, the tree $T_{z}$ is wellfounded. Let $M_{z}=\overline{T \cup R}$, where $T$ is the union of all 2-dimensional 
tubes in the framework and $R$ is the union of all adjoined regions. Clearly, $(0,0, z) \in M_{z}$. We claim that $M_{z}$ is a Cantor 2-manifold, which completes the proof.

By way of contradiction, assume there is a 0-dimensional set $I \subset M_{z}$ such that $M_{z} \backslash I=U \cup V$, where $U$ and $V$ are nonempty, disjoint and relatively open sets in $M_{z} \backslash I$.

Lemma 3. There exist a pair of "consecutive" tubes, i.e., tubes of the form $T\left(i_{1}, \ldots, i_{m-1}, i_{m}\right)$ and $T\left(i_{1}, \ldots, i_{m-1}, i_{m}+1\right)$, such that $T\left(i_{1}, \ldots\right.$ $\left.\ldots, i_{m-1}, i_{m}\right) \backslash I \subset U$ and $T\left(i_{1}, \ldots, i_{m-1}, i_{m}+1\right) \backslash I \subset V$ or vice versa.

Proof. It is easy to see that each of $U$ and $V$ must contain a tube $(\bmod I)$, and the tubes are partitioned accorded to whether they are a subset of $U$ or of $V(\bmod I)$. Suppose that the lemma fails and let $T\left(i_{1}, \ldots, i_{m}\right)$ be a tube in $U$ (more precisely, $\left.T\left(i_{1}, \ldots, i_{m}\right) \backslash I \subset U\right)$. Since the lemma fails, we have $T\left(i_{1}, \ldots, i_{m-1}, j\right) \backslash I \subset U$, for all $j \geq 1$. This then implies that $T\left(i_{1}, \ldots, i_{m-1}+1\right) \backslash I \subset U$. Likewise, we then get $T\left(i_{1}, \ldots, i_{m-2}+1\right) \backslash I \subset$ $U, \ldots, T\left(i_{1}+1\right) \backslash I \subset U$. Similarly, starting with a tube contained in $V$, we get a tube $T\left(j_{1}+1\right)$ such that $T\left(j_{1}+1\right) \backslash I \subset V$. This easily gives the lemma.

Assume now, without loss of generality, that $T\left(i_{1}, \ldots, i_{m}\right) \backslash I \subset U$ and $T\left(i_{1}, \ldots, i_{m}+1\right) \backslash I \subset V$. Since $T\left(i_{1}, \ldots, i_{m}+1\right) \backslash I \subset V$, it follows that $T\left(i_{1}, \ldots, i_{m}, i_{m+1}\right) \backslash I \subset V$, for some $i_{m+1} \geq 1$ (in fact for all sufficiently large $\left.i_{m+1}\right)$. From this we easily get consecutive tubes $T\left(i_{1}, \ldots, i_{m}, i_{m+1}\right) \backslash$ $I \subset U$ and $T\left(i_{1}, \ldots, i_{m+1}+1\right) \backslash I \subset V$ (note that we may have $i_{m+1}=0$ ). Continuing, we define a sequence $\left(i_{1}, i_{2}, \ldots\right) \in \omega^{\omega}$. Since $T_{z}$ is well-founded, some $\left(f\left(i_{1}\right), \ldots, f\left(i_{k}\right)\right) \notin T_{z}$ with $k \geq m$, and thus $R\left(i_{1}, \ldots, i_{k}\right) \subset M_{z}$. This is impossible, since $T\left(i_{1}, \ldots, i_{k}\right) \backslash I \subset U, T\left(i_{1}, \ldots, i_{k}+1\right) \backslash I \subset V$, and $\left.T\left(i_{1}, \ldots, i_{k}\right) \cup T\left(i_{1}, \ldots, i_{k}+1\right) \cup R\left(i_{1}, \ldots, i_{k}\right)\right) \backslash I$ is connected. This establishes part (b) of the theorem except for the fact that $K(X)$ is $\boldsymbol{\Pi}_{1}^{1}$. A straightforward computation shows that $P$ is the complicated part of $K(X)$; the part of $K(X)$ not on the $z$-axis is an $F_{\sigma}$ set.

Pro of of (c). We work now in $\mathbb{R}^{4}$. We will use the construction given in the proof of part (b). Let $C$ be the Cantor middle third set (we again will think of $C$ as $\left.\{0,1\}^{\omega}\right)$. Let $P \subset C$ be $\Sigma_{2}^{1}$. We define a compact subset $X$ of $\mathbb{R}^{4}$ such that for $z \in C,(0,0, z, z) \in K(X) \leftrightarrow z \in P$. This will certainly show $K(X)$ may be $\Sigma_{2}^{1}$ complete.

Since $P$ is $\boldsymbol{\Sigma}_{2}^{1}$, we have $P(z) \leftrightarrow H(z, w)$ where $H \subset\{0,1\}^{\omega} \times\{0,1\}^{\omega}$ is $\boldsymbol{\Pi}_{1}^{1}$. The idea is to associate with each $z$ in $C$ a collection of triangular regions, one for each $w \in\{0,1\}^{\omega}$, with common point $(0,0, z, z)$. Each such triangular region $T_{z, w}$ is filled in exactly as in part (b), using now the pair $(z, w)$ instead of $z$. Thus, we might envision such a set as in Fig. 2. 


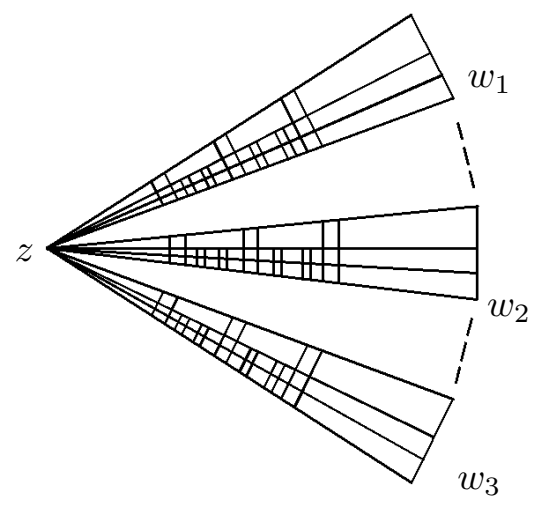

Fig. 2

We run into a problem trying to carry out this construction in $\mathbb{R}^{3}$, however, as we cannot guarantee that the sets $T_{z, w}$ corresponding to different $z$ 's will be disjoint. We found it necessary to add one more dimension to assure this last disjointness property holds.

To be specific, for each $z, w \in\{0,1\}^{\omega}$, we define a "triangle" in $\mathbb{R}^{4}, T_{z, w}$, to be the union of the closed line segments joining the points $(0,0, z, z)$, $(1,1, w, z)$ and $(-1,1, w, z)$. For each such $(z, w) \in\{0,1\}^{\omega} \times\{0,1\}^{\omega}$, we define $E_{z, w}$ to be the "filled-in" version of $T_{z, w}$ exactly as $X_{z}$ was the filling of $D_{z}$ in case (b). We then set $X=\bigcup_{z, w} E_{z, w}$.

An argument analogous to that given in part (b) can be given to show that $X$ is closed. Also, note that $X$ is still 2-dimensional, since it is a subset of $\mathbb{R}^{2} \times C \times C$.

Also, if $z \in P$, then for some $w \in\{0,1\}^{\omega},(z, w) \in H$. But then, arguing exactly as in case (b), $M_{z, w}$ is a Cantor 2-manifold containing $(0,0, z, z)$. Thus, $(0,0, z, z) \in K(X)$.

Finally, suppose $z \notin P$. So, for all $w \in\{0,1\}^{\omega},(z, w) \notin H$. We show that $(0,0, z, z) \notin K(X)$. Suppose to the contrary that $M$ were a Cantor 2-manifold with $(0,0, z, z) \in M \subset X$. If for some $w \in\{0,1\}^{\omega}$, we have $M \subset E_{z, w}$, then we would have a contradiction exactly as in (b). Thus, for some $\left(z^{\prime}, w^{\prime}\right) \neq(z, w), M \cap E_{z, w} \neq \emptyset$ and $M \cap E_{z^{\prime}, w^{\prime}} \neq \emptyset$. If $z \neq z^{\prime}$, we have a contradiction as we may define a separation of $M$ by $U=M \cap\{(a, b, c, d)$ : $d>s\}, V=M \cap\{(a, b, c, d): d<s\}$, where $s$ is between $z$ and $z^{\prime}$ and $s \notin C$. So, we may assume $M \subset \bigcup_{w} E_{z, w}$ and $M \cap E_{z, w} \nsubseteq\{\{(0,0, z, z)\}$ and $M \cap E_{z, w^{\prime}} \nsubseteq\{(0,0, z, z)\}$ for some $w \neq w^{\prime}$. However, we may then clearly separate $M \backslash\{(0,0, z, z)\}$, a contradiction. This completes the proof of Theorem 2.

As we remarked, the construction for part (c) of the theorem seems to require working with $\mathbb{R}^{4}$. 
Question 1. If $X \subset \mathbb{R}^{3}$, is $K(X)$ always $\boldsymbol{\Pi}_{1}^{1}$ ?

QUESTION 2. Simply from the general theory of projective sets we know there is some $\boldsymbol{\Sigma}_{2}^{1}$ norm (or $\boldsymbol{\Sigma}_{2}^{1}$ operator) on $X$ which yields $K(X)$. It would be very interesting to give some natural topological definition of this norm or operator. Perhaps such a norm or operator would be $\boldsymbol{\Pi}_{1}^{1}$ for continua in $\mathbb{R}^{3}$. This would give a positive solution to our Question 1 .

Question 3. Is there an $n$-dimensional compactum $X$ such that $K(X)$ is $\Sigma_{2}^{1}$ complete and $X \backslash K(X)$ is also $n$-dimensional? We conjecture the answer to this question is yes. If so, can $X$ be taken to be homogeneous?

QUESTION 4. Is there a continuum $M$ such that the homeomorphism group $X$ of $M$ is $n$-dimensional and $X \backslash K(X)$ is $\boldsymbol{\Sigma}_{2}^{1}$ complete? (We note that the existence of totally disconnected homeomorphism groups of dimension $n$ is not completely settled $[\mathrm{B}, \mathrm{M}]$.)

II. A result about Lebesgue measure. A real-valued function $f$ defined on a complete separable metric space $X$ is said to be countably continuous provided $X$ can be partitioned into countably many disjoint Borel sets $B_{1}, B_{2}, \ldots$ such that for each $i$, the partial function $f \mid B_{i}$ is continuous on $B_{i}$. Clearly, every continuous function is countably continuous and there are countably continuous Borel measurable functions of arbitrarily high Borel class.

Note. In this definition of countably continuous we required the sets $B_{1}, B_{2}, \ldots$ to be Borel sets. However, this is no restriction within the class of Borel measurable functions, in view of the fact that a Borel measurable function $f$ is countably continuous in our sense if and only if there is a partition of $X$ into sets $C_{1}, C_{2}, \ldots$ such that $f \mid C_{i}$ is continuous on $C_{i}$. To prove this equivalence one uses the fact that if $f$ is continuous on $E \subset X$, then there is a Borel set $\widehat{E} \supset E$ and a continuous extension $\widehat{f}$ of $f$ to $\widehat{E}$.

Recall that $f$ is lower semicontinuous if $f$ is the pointwise supremum of a sequence of continuous functions. Adyan and Novikov showed there exists a lower semicontinuous function which is not countably continuous $[\mathrm{A}-\mathrm{N}]$. We reprove this result here. We then give an explicit example by showing that Lebesgue measure regarded as a function on the space of compact subsets of $[0,1]$, which is upper semicontinuous, is not countably continuous.

We first provide a quick proof of the result of Adyan and Novikov which illustrates our proof for Lebesgue measure.

TheOREM 4. Let $X$ be an uncountable complete separable metric space. Then there is a lower semicontinuous $f: X \rightarrow \mathbb{R}$ which is not countably continuous. 
Proof. Fix a $\Sigma_{1}^{0}$ set $U \subset X \times \omega$ which is universal in the sense that for each $x \in X, U_{x}=\{n:(x, n) \in U\}$ is a complete $\Sigma_{1}^{0}(x)$ set. For each $x \in X$, let $f(x) \in \mathbb{R}$ be defined by $f(x)=\sum_{n=0}^{\infty} 3^{-n} a_{n}$, where $a_{n}=1$ if $n \in U_{x}$ and $a_{n}=0$ if $n \notin U_{x}$.

First, note that $f$ is lower semicontinuous. To see this, write $U(n, x) \leftrightarrow$ $\exists m R(x, m, n)$, where $R \subset X \times \omega \times \omega$ is recursive. Then, for each $k \in \omega$, define $f_{k}: X \rightarrow \mathbb{R}$ by $f_{k}(x)=\sum_{n=0}^{k} 3^{-n} a_{n, k}$, where $a_{n, k}=1$ if $\exists n \leq k$ $R(x, m, n)$ and 0 otherwise. Clearly, $f=\sup _{k} f_{k}$, and each $f_{k}$ is continuous (in fact recursive).

Suppose that $f$ were countably continuous, and let $\alpha_{1}, \alpha_{2} \ldots \in X$ code Borel sets $B_{1}, B_{2}, \ldots$ which have disjoint union $X$ and such that $f \mid B_{i}$ is continuous for each $i$. Since $f \mid B_{i}$ is continuous, there is a "real" $\beta_{i} \in X$ providing a code for $f \mid B_{i}$; i.e., the relation $\{(s, t): s, t \in \omega, s, t$ code basic neighborhoods $N_{s}, N_{t}$ of $x$ such that $\left.f\left(B_{i} \cap N_{s}\right) \subset N_{t}\right\}$ is $\Delta_{1}^{0}\left(\beta_{i}\right)$. Let $\gamma=\left\langle\alpha_{1}, \ldots, \alpha_{n}, \beta_{1}, \ldots, \beta_{n}\right\rangle$. Then $\gamma \in B_{i}$ for some $i$, and thus, $f(\gamma) \in$ $\Delta_{1}^{0}\left(\gamma, \beta_{i}\right)=\Delta_{1}^{0}(\gamma)$. This contradicts the fact that $f(\gamma)$ is $\Sigma_{1}^{0}(\gamma)$ complete.

We turn now to the corresponding question for Lebesgue measure. We now let $Y$ denote the compact metric space $\{0,1\}^{\omega}$ with Lebesgue or Haar measure, $\mu$. This is for convenience only as the proof below will work with minor modification for $Y=[0,1]$ and the usual Lebesgue measure.

TheOrem 5. Let $X$ denote the space of closed subsets of $Y$ with the Hausdorff metric. Then $\mu: X \rightarrow \mathbb{R}$ is upper semicontinuous but is not countably continuous.

P r o of. It is well known that $\mu$ is upper semicontinuous. Let us assume that $\mu$ is countably continuous. Following the outline of the above argument, it is enough to show that for any $\gamma^{\prime} \in Y=\{0,1\}^{\omega}$, there is a closed $F \subset Y$ such that if $\gamma \in Y$ is the real coding $F$ (i.e., $\gamma$ codes the tree $T \subset\{0,1\}<\omega$ representing $F$ ), then $\gamma={ }_{\mathrm{T}} \gamma^{\prime}$ and $\mu(F) \notin \Delta_{1}^{0}(\gamma)$, where $\leq_{\mathrm{T}}$ denotes Turing equivalence. Since the argument below works uniformly in any degree, we may now take $\gamma^{\prime}={ }_{\mathrm{T}} 0$. That is, we produce an $F \subset Y$ with recursive code but such that $\mu(F)$ is not recursive.

To do this, let $A \subset \omega$ be a $\Sigma_{1}^{0}$ complete set, so $A(n) \leftrightarrow \exists m R(m, n)$ where $R \subset \omega \times \omega$ is recursive. Define a tree $T \subset\{0,1\}^{<\omega}$ by $T(s) \leftrightarrow s \in\{0,1\}^{<\omega}$ and $s=t^{\wedge} 0^{\wedge} u$ where $t$ is a sequence of 1 's of even length $2 j, u \in\{0,1\}^{<\omega}$, and $\forall k \leq$ length $(u)[\exists m \leq k R(j, m) \rightarrow u(k)=1]$. Clearly $T$ can be coded by a recursive real $\gamma$. Let $F \subset Y$ be the closed set corresponding to $T$. We claim that $\mu(F)$ is not recursive. For suppose $\mu(F)$ were recursive. Then we could show $A$ is recursive as follows: Given $n \in \omega$, consider all possible sums of the form $s=\sum_{p=0}^{n-1} \varepsilon_{p}(1 / 4)^{p+1}$ where $\varepsilon_{p}=0$ or 1 . For each of these, determine if $|\mu(F)-s|<1 / 4^{n+2}$. If any of the $s$ satisfy this inequality, then 
$n \in A$, otherwise $n \notin A$. This follows since $\mu(F)=\sum_{p=0}^{\infty} \varepsilon_{p}(1 / 4)^{p+1}$ where $\varepsilon_{p}=1$ if $p \notin A$ and is 0 otherwise. Since $\mu(F)$ was assumed recursive, this is a recursive procedure, violating the assumption that $A$ is $\Sigma_{1}^{0}$ complete. This completes the proof of the theorem.

QUESTION 5. We conjecture that almost all derivatives are not countably continuous. Consider the Banach space of all bounded derivatives on $[0,1]$. Is it true that the set of functions in this space which are countably continuous is meager?

In fact, we conjecture the same is true for functions in Baire class 1:

Question 6. Consider the Banach space of all bounded Baire class 1 functions on $[0,1]$. Is it true that the set of functions in this space which are countably continuous is meager?

Added in proof. Results of related interest concerning countable continuity have been obtained by J. Cichon, M. Morayne, J. Pawlikowski and S. Solecki, Decomposing Baire functions, J. Symbolic Logic 56 (1991), 1273-1283.

\section{References}

[A] P. S. Aleksandrov, On the dimension of closed sets, Uspekhi Mat. Nauk 4 (6) (1949), 17-88 (in Russian).

[A-N] S. I. Adyan and P. S. Novikov, On a semicontinuous function, Moskov. Gos. Ped. Inst. Uchen. Zap. 138 (3) (1958), 3-10 (in Russian).

[B] B. L. Brechner, On the dimensions of certain spaces of homeomorphisms, Trans. Amer. Math. Soc. 121 (1966), 516-548.

[E] R. Engelking, Dimension Theory, PWN and North-Holland, Warszawa-Amsterdam 1978.

[M] J. van Mill, n-dimensional totally disconnected topological groups, Math. Japon. 32 (1987), 267-273.

[P] R. Pol, An n-dimensional compactum which remains $n$-dimensional after removing all Cantor n-manifolds, Fund. Math. 136 (1990), 127-131.

DEPARTMENT OF MATHEMATICS

UNIVERSITY OF NORTH TEXAS

DENTON, TEXAS 76203

U.S.A. 